\title{
Supporting Information for \\ Dehydration of Cations Inducing Fast Ion Transfer and High Electrical Capacitance Performance on Graphene Electrode in Aqueous Electrolytes
}

Sharifa Faraezi, Md Sharif Khan, and Tomonori Ohba*

${ }^{\dagger}$ Graduate School of Science, Chiba University, 1-33 Yayoi, Inage, Chiba 263-8522, Japan

Corresponding Author

*E-mail: ohba@chiba-u.jp.

Experimental and Simulation Procedures

Table S1

Figures S1-S7

References 


\section{Experimental and Simulation Procedures}

Experimental Diffusion. The critical scan rate is defined as that at the intersection of two lines in the regions of fast and slow scan rates. Here, the critical scan rates are denoted by $v_{c}$ and were calculated from the relationship between the charges and scan rates from 0.1 to $1000 \mathrm{mV} \mathrm{s}^{-1}$, as shown in Figure S5. The perspective diffusion coefficient was calculated by the equation:

$$
D=\frac{h^{2}}{\tau}, \tau=\frac{V_{w}}{v_{c}}
$$

The diffusion coefficient $(D)$ was determined by dividing the thickness of the electrode material $(h)$ to the time constant $(\tau) .{ }^{1}$ Here, $V_{w}$ and $v_{\mathrm{c}}$ are the total potential window of the cyclic voltammetry and the critical scan rate, respectively. The relative diffusion coefficient $\left(D_{\mathrm{r}}\right)$ was defined by neglecting $h$,

$D_{r}=\frac{D}{h^{2}}=\frac{1}{\tau}$

The relative diffusion coefficient was directly compared in the different electrolyte solutions.

Molecular Dynamics (MD) Simulations. MD simulations were performed using the large-scale Atomic/Molecular Massively Parallel Simulator molecular dynamics code. Topological files were built with Packmol and Moltemplate. ${ }^{2-3}$ The particle mesh Ewald sum was used to perform long ranged Coulomb interactions with a real space cutoff of 
$1.2 \mathrm{~nm}$. The cutoff length for the van der Waals interaction was $1.0 \mathrm{~nm}$. The single sheet graphene surface consisted with 416 rigid atoms, which were fixed to their initial positions. The 12-6 Lennard-Jones potential parameters of graphene were $\sigma_{\mathrm{G}}=0.34 \mathrm{~nm}$ and $\varepsilon_{\mathrm{G}}=2.3263 \mathrm{kJmol}^{-1}$ with a C-C distance $0.142 \mathrm{~nm} .{ }^{4}$ The TIP/3P model of water was used with parameters of $\sigma_{\mathrm{o}}=0.3188 \mathrm{~nm}$ and $\varepsilon_{\mathrm{o}}=0.4268 \mathrm{~kJ} \mathrm{~mol}^{-1}{ }^{5}$ The SHAKE algorithm was used to hold the two $\mathrm{O}-\mathrm{H}$ bonds and the $\mathrm{H}-\mathrm{O}-\mathrm{H}$ angle rigid. ${ }^{6}$ The $\mathrm{LJ}$ potential parameters of $\mathrm{K}^{+}, \mathrm{Na}^{+}, \mathrm{Li}^{+}, \mathrm{Br}^{-}, \mathrm{Cl}^{-}$, and $\mathrm{F}^{-}$were $\sigma_{\mathrm{K}+}=0.3143 \mathrm{~nm}, \varepsilon_{\mathrm{K}+}=$ $0.363757 \mathrm{~kJ} \mathrm{~mol}^{-1}, \sigma_{\mathrm{Na}^{+}}=0.2430 \mathrm{~nm}, \varepsilon_{\mathrm{Na}^{+}}=0.195853 \mathrm{~kJ} \mathrm{~mol}^{-1}, \sigma_{\mathrm{Li}^{+}}=0.2126 \mathrm{~nm}, \varepsilon_{\mathrm{Li}+}$ $=0.0764835 \mathrm{~kJ} \mathrm{~mol}^{-1}, \sigma_{\mathrm{Br}-}=0.3970 \mathrm{~nm}, \varepsilon_{\mathrm{Br}-}=0.205545 \mathrm{~kJ} \mathrm{~mol}^{-1} \sigma_{\mathrm{Cl}-}=0.4045 \mathrm{~nm}, \varepsilon_{\mathrm{Cl}-}$ $=0.627182 \mathrm{~kJ} \mathrm{~mol}^{-1}$, and $\sigma_{\mathrm{F}-}=0.3118 \mathrm{~nm}, \varepsilon_{\mathrm{F}-}=0.75354 \mathrm{~kJ} \mathrm{~mol}^{-1}$, respectively. ${ }^{7-9} \mathrm{The}$ validity of the potential parameters was discussed in the preceding reports. ${ }^{7}$ Standard electrostatic change was considered for the ions. The Lorentz-Berthelot mixing rules were adopted for all mixing Lennard-Jones interactions. As depicted in figure S1, 1200 $\mathrm{H}_{2} \mathrm{O}$ molecules and 21 cations and anions were considered for a $1.0 \mathrm{M}$ concentration in a $3.07 \times 3.07 \times 4.0 \mathrm{~nm}^{3}$ unit cell; however, the periodic boundary condition was changed to be $3.07 \times 3.07 \times 6.0 \mathrm{~nm}^{3}$ to avoid passage of molecules to the opposite site of the graphene while the graphene was charge neutral. The concentration in those simulations was different from $0.1 \mathrm{M}$ experimental concentration because there were a very few ions approximately only one ion present in the simulation box when a $1.0 \mathrm{M}$ concentration in the simulated box was assumed. During charging (positive and negative) of graphene, anion and cation numbers were controlled by preserving the charge balance among the ions and charged carbon atoms on graphene. Here, $\pm 5.00 \mathrm{e}$ on graphene, i.e., \pm 0.012019 e per a carbon atom, corresponded to $\pm 0.4 \mathrm{~V}$ in the experiments. Since the theoretical intrinsic capacitance of graphene is $21 \mu \mathrm{F} \mathrm{cm}^{-2}$ and the maximum total charge of $11.7 \mathrm{e}$ 
on graphene sheet $(3 \mathrm{~nm} \times 3 \mathrm{~nm})$ for $1 \mathrm{~V}$ potential. For our $0.4 \mathrm{~V}$ system, calculated total charge was $4.68(\sim 5.00 \mathrm{e})$ and divided by carbon numbers as graphene sheet has 416 carbon atoms. ${ }^{8}$ Here the partial charges of carbon atoms were assumed to correspond to the intrinsic capacitance. The adsorption amounts of cation and anion were calculated from the numbers of ions shorter distances than the valley between the first and second peaks from graphene interfaces in the density distributions shown in Figure $3 b$. The adsorption number of ions were evaluated from the averaging of last $0.5 \mathrm{~ns}$ simulation data. A homogeneous distribution of the applied charge over all the carbon atoms was assumed during the simulation. All systems were simulated for $1 \mathrm{~ns}$ first with an NPT ensemble at 1 atm to determine the bulk like properties of electrolytes. The NVT ensemble simulation was subsequently used for 2 ns with a time step of $1.5 \mathrm{fs}$, providing an equilibrium structure of electrolyte solution on graphene interfaces. The Nosé-Hoover algorithm was used to maintain the temperature at 298K. The last $1 \mathrm{~ns}$ were used to evaluate the ion dynamics and hydration structure. The theoretical selfdiffusion coefficient was measured from the following equation,

$D=\frac{\left\langle R(t)-R(0)^{2}\right\rangle}{6 t}$

where $D$ is the self-diffusion coefficient and $R(t)$ is the position vector of atoms/ions at time $t$. 
Table S1 Ionic radius, ionic mobilities and ionic conductivity of aqueous electrolyte ions at $298 \mathrm{~K}^{1,10-12}$

\begin{tabular}{ccc}
\hline Ion Species & Ionic radius $/ \mathrm{nm}$ & Ionic mobility $/ 10^{-8} \mathrm{~m}^{2} \mathrm{~s}^{-1} \mathrm{~V}^{-1}$ \\
\hline $\mathrm{Li}^{+}$ & 0.059 & 4.01 \\
$\mathrm{Na}^{+}$ & 0.102 & 5.19 \\
$\mathrm{~K}^{+}$ & 0.138 & 7.62 \\
$\mathrm{~F}^{-}$ & 0.133 & 5.74 \\
$\mathrm{Cl}^{-}$ & 0.181 & 7.91 \\
$\mathrm{Br}^{-}$ & 0.195 & 8.09 \\
\hline
\end{tabular}

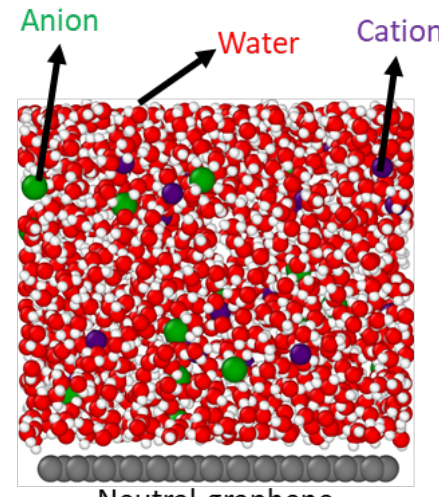

Neutral graphene

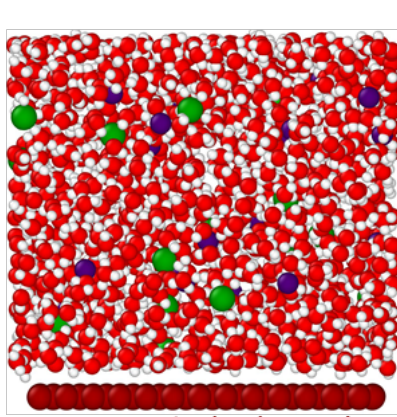

Negatively charged graphene

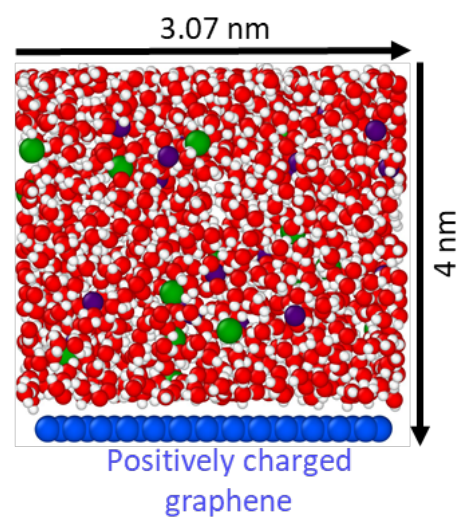

graphene

Figure S1. Initial setup for simulation diagram of an electrolyte system with neutral (grey) and negatively (red) and positively charged (blue) graphene surfaces. 


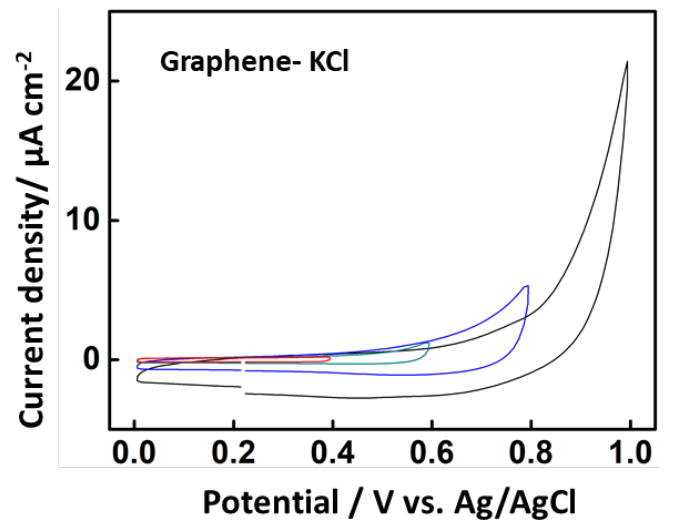

Figure S2. Cyclic voltammetric profile recorded in $0.1 \mathrm{M} \mathrm{KCl}$ electrolyte using singlelayer graphene coated Au electrode in the potential ranges of $0.0-0.4 \mathrm{~V}$ (red), 0.0-0.6 V (green), 0.0-0.8 V (blue), and 0.0-1.0 V (black curve). Scan rate was set to $100 \mathrm{mV} \mathrm{s}^{-1}$.

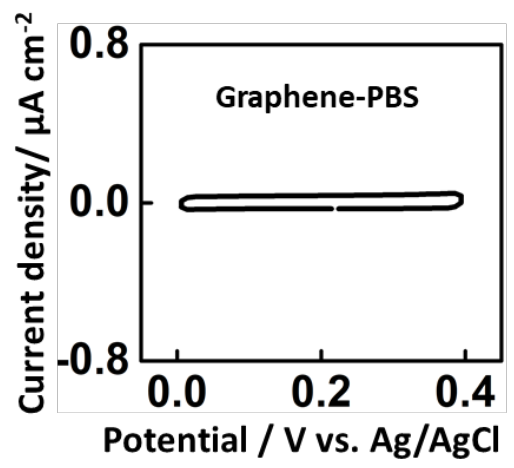

Figure S3. Cyclic voltammogram obtained on graphene electrodes in phosphate buffer solution ( $\mathrm{pH} 7)$ at a scan rate $100 \mathrm{mV} \mathrm{s}^{-1}$. 
(a)
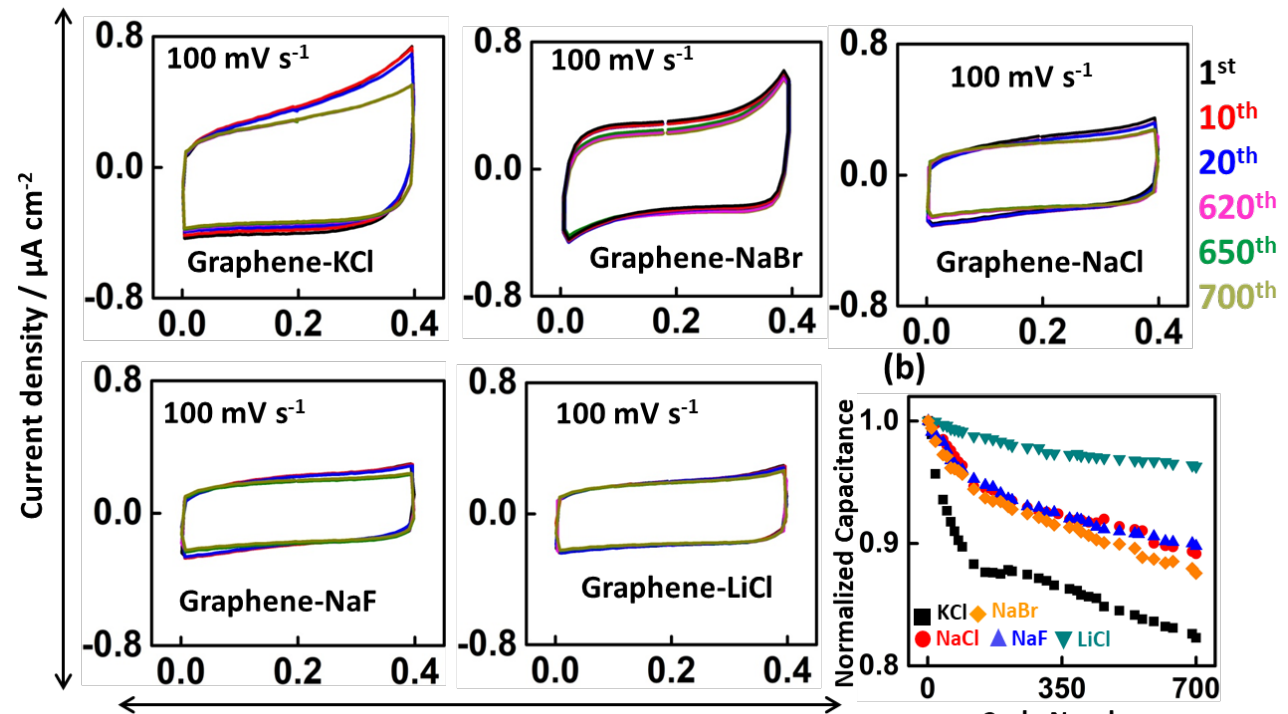

(b)

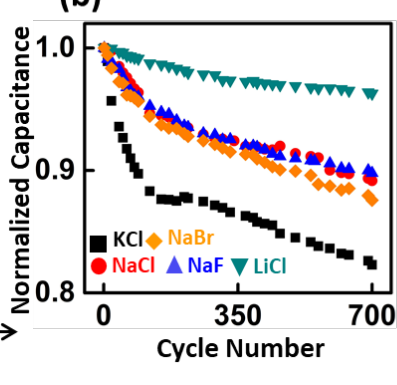

Figure S4. (a) Cyclic voltammograms of graphene electrodes in $\mathrm{KCl}, \mathrm{NaBr}, \mathrm{NaCl}, \mathrm{NaF}$, and $\mathrm{LiCl}$ electrolyte solutions after 1, 10, 20, 620, 650, and 700 cycles. (b) Normalized capacitance of different electrolytes at scan rate $100 \mathrm{mV} \mathrm{s}^{-1}$ of graphene with continuous charge/discharge cycling for $\mathrm{KCl}(\boldsymbol{\square}), \mathrm{NaBr}(\boldsymbol{\vee}), \mathrm{NaCl}(\boldsymbol{O}), \mathrm{NaF}(\boldsymbol{\Delta})$, and $\mathrm{LiCl}(\boldsymbol{\nabla})$.

(a)

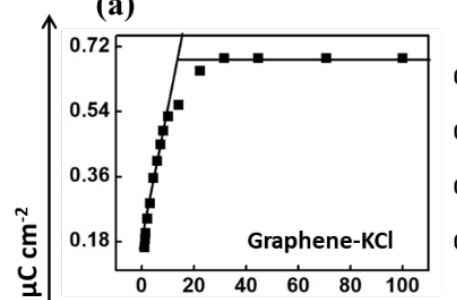

(b)

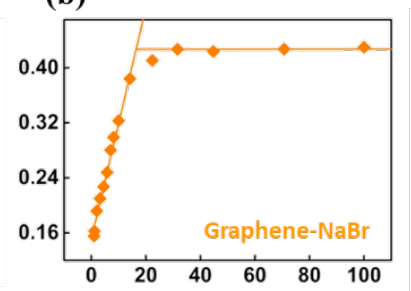

(c)

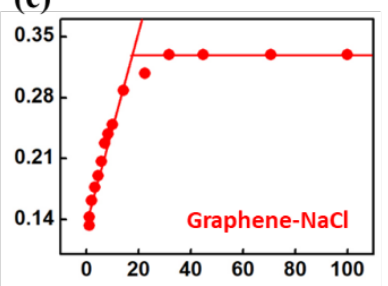

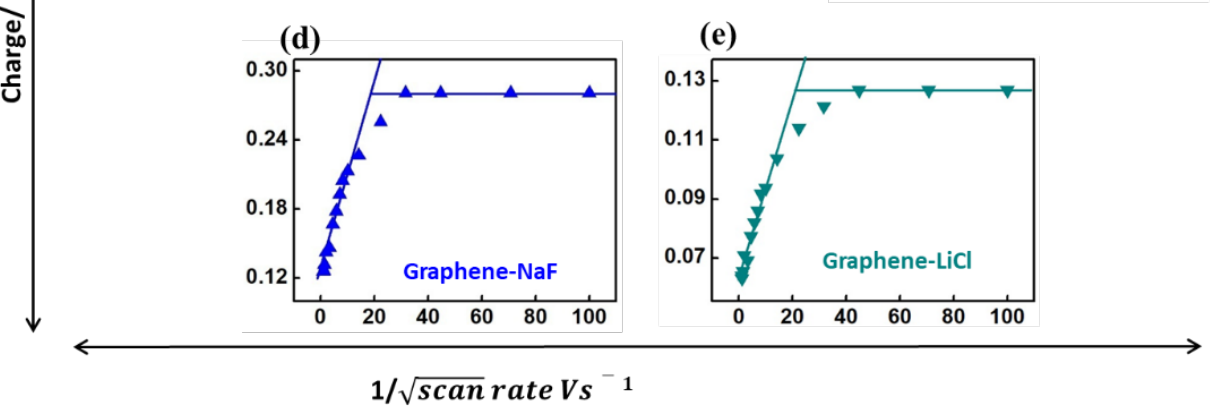

Figure S5. Critical scan rates determined from charge vs. inverse square root of scan rates on graphene electrodes. Critical scan rates of electrolyte solutions in graphene system as estimated from the crossing point of the two linear lines on the graphs. 


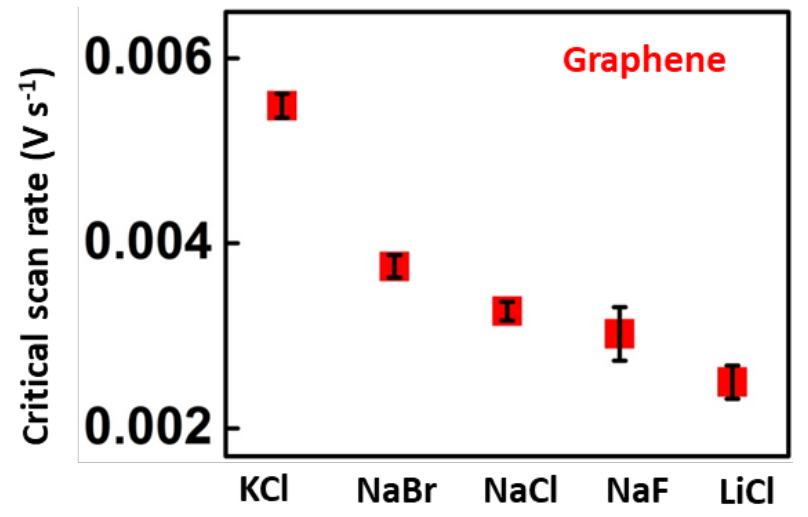

Figure S6. Critical scan rates for $\mathrm{KCl}, \mathrm{NaBr}, \mathrm{NaCl}, \mathrm{NaF}$, and $\mathrm{LiCl}$ electrolyte solutions on graphene surfaces.

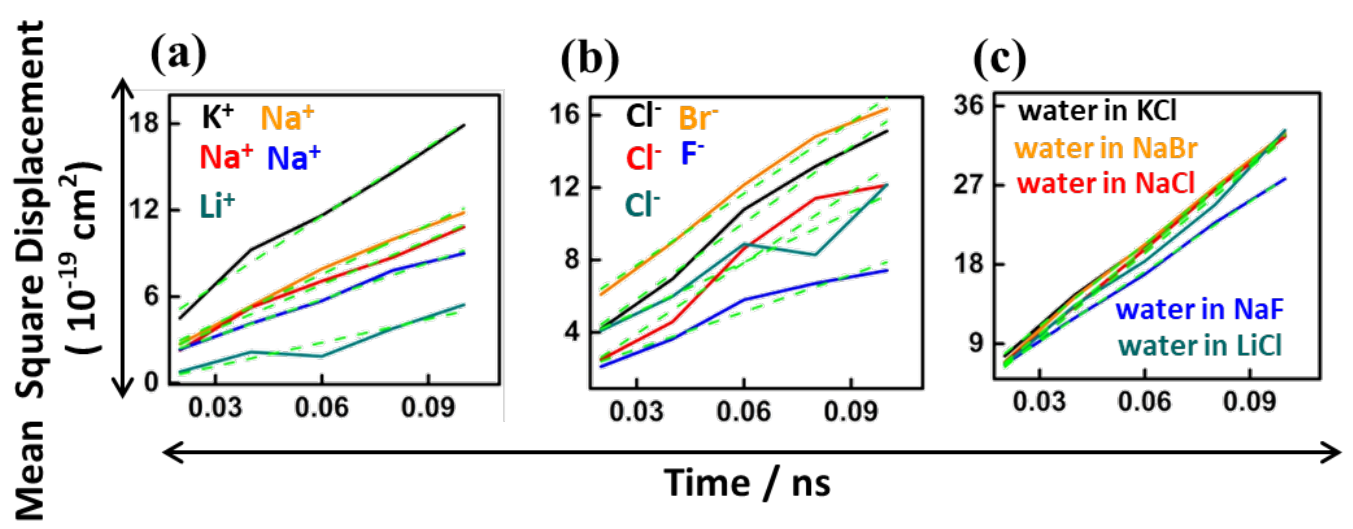

Figure S7. Means square displacement of (a) cations, (b) anions, and (c) water molecules in electrolytes, respectively. 


\section{References}

(1) Zhu, J.; Xu, Y.; Wang, J.; Lin, J.; Sun, X.; Mao, S., The Effect of Various Electrolyte Cations on Electrochemical Performance of Polypyrrole/ Rgo Based Supercapacitors. Phys. Chem. Chem. Phys. 2015, 17, 28666.

(2) Martínez, L.; Andrade, R.; Birgin, E. G.; Martínez, J. M., Packmol: A Package for Building Initial Configurations for Molecular Dynamics Simulations. J. Comput. Chem. 2009, 30, 2157-2164.

(3) Martínez, O. M.; Ne, L. M., Packing Optimization for Automated Generation Ofcomplex System'S Initial Configurations for Molecular Dynamics and Docking. J. Comput. Chem. 2003, 24, 819-825.

(4) Ohba, T., The Thinnest Molecular Separation Sheet by Graphene Gates of Single-Walled Carbon Nanohorns. ACS Nano 2014, 8, 11313-11319.

(5) Price, D. J.; Brooks, C. L., A Modified Tip3p Water Potential for Simulation with Ewald Summation. J. Chem. Phys. 2004, 121, 10096-103.

(6) Ryckaert, J.-P.; Ciccotti, G.; Berendsen, H. J. C., Numerical Integration of the Cartesian Equations of Motion of a System with Constraints: Molecular Dynamics of $\mathrm{N}$ Alkanes. J. Comput. Phys 1977, 23, 327.

(7) Joung, I. S.; Cheatham, T. E., Determination of Alkali and Halide Monovalent Ion Parameters for Use in Explicitly Solvated Biomolecular Simulations. J. Phys. Chem. B 2008, 112, 9020-9041.

(8) Jiang, G.; Cheng, C.; Li, D.; Liu, J. Z., Molecular Dynamics Simulations of the Electric Double Layer Capacitance of Graphene Electrodes in Mono-Valent Aqueous Electrolytes. Nano Res. 2016, 9, 174-186.

(9) Canongia Lopes, J. N.; Pádua, A. A. H., Molecular Force Field for Ionic Liquids lii: Imidazolium, Pyridinium, and Phosphonium Cations; Chloride, Bromide, and Dicyanamide Anions. J. Phys. Chem. B 2006, 110, 19586-19592.

(10) Richards, L. A.; Richards, B. S.; Corry, B.; Schäfer, A. I., Experimental Energy Barriers to Anions Transporting through Nanofiltration Membranes. Environ. Sci. Technol. 2013, 47, 1968-1976.

(11) Richards, L. A.; Richards, B. S.; Corry, B.; Schafer, A. I., Response to Comment on " Experimental Energy Barriers to Anions Transporting Though Nanofiltration Membranes". Environ. Sci. Technol. 2013, 47, 8987-8988.

(12) Owen, B. B.; Zeldes, H., The Conductance of Potassium Chloride, Potassium Bromide and Potassium lodide in Aqueous Solutions from 5 to $55^{\circ}$. J. Chem. Phys. 1950, 18, 1083-1085. 